\section{Comparison of Air-Gaps Effect in a Small Cavity on Dose Calculation for 6 MV Linac}

\author{
Azzi A. ${ }^{1 \oplus}$, Ryangga D. ${ }^{2}$, Pawiro S. A. ${ }^{3 * \odot}$
}

\begin{abstract}
Background: Many authors stated that cavities or air-gaps were the main challenge of dose calculation for head and neck with flattening filter medical linear accelerator (Linac) irradiation.
\end{abstract}

Objective: The study aimed to evaluate the effect of air-gap dose calculation on flattening-filter-free (FFF) small field irradiation.

Material and Methods: In this comparative study, we did the experimental and Monte Carlo (MC) simulation to evaluate the presence of heterogeneities in radiotherapy. We simulated the dose distribution on virtual phantom and the patient's CT image to determine the air-gap effect of open small field and modulated photon beam, respectively. The dose ratio of air-gaps to tissue-equivalent was calculated both in Analytical Anisotropic Algorithm (AAA) and MC.

Results: We found that the dose ratio of air to tissue-equivalent tends to decrease with a larger field size. This correlation was linear with a slope of $-0.198 \pm 0.001$ and $-0.161 \pm 0.014$ for both AAA and MC, respectively. On the other hand, the dose ratio below the air-gap was field size-dependent. The AAA to MC dose calculation as the impact of air-gap thickness and field size varied from 1.57\% to 5.35\% after the gap. Besides, patient's skin and oral cavity on head and neck case received a large dose discrepancy according to this study.

Conclusion: The dose air to tissue-equivalent ratio decreased with smaller air gaps and larger field sizes. Dose correction for AAA calculation of open small field size should be considered after small air-gaps. However, delivered beam from others gantry angle reduced this effect on clinical case.

Citation: Azzi A, Ryangga D, Pawiro SA. Comparison of Air-Gaps Effect in a Small Cavity on Dose Calculation for 6 MV Linac. J Biomed Phys Eng. 2021;11(1):17-28. doi: 10.31661/jbpe.v0i0.2004-1096.

\section{Keywords}

Cancer; Therapeutics; Radiotherapy; Dosage; Monte Carlo Method

\section{Introduction}

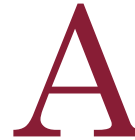

dvanced radiotherapy techniques such as Intensity Modulated Radiation Therapy (IMRT), Volumetric Modulated Arc Therapy (VMAT), and Stereotactic Radiotherapy (SRT) are widely implemented to treat cancer patients. Recent technology developments have shown that the removal of the flattening filter from the head Linac is advantageous for advanced irradiation techniques. However, the removal of the flattening filter affects the characteristics of the Linac photon output, as the flattening filter is known to be the main attenuator component. On the other hand, the algorithms of the treatment planning system (TPS) have been optimized for flattened beams. In a flattening filter free (FFF) photon beam, the beam model needs to be changed.
${ }^{1} \mathrm{MSc}$, Department of

Physics, Faculty of Math-

ematics and Natural

Sciences, University of

Indonesia, Depok, West

Java, 16424, Indonesia

${ }^{2} \mathrm{MSc}$, Department of

Radiotherapy, Pasar

Minggu Regional Gen-

eral Hospital, South

Jakarta, Jakarta, 12550

Indonesia

${ }^{3} \mathrm{PhD}$, Department of

Physics, Faculty of Math-

ematics and Natural

Sciences, University of

Indonesia, Depok, West

Java, 16424, Indonesia

*Corresponding author:

S. A. Pawiro

Department of Physics,

Faculty of Mathematics

and Natural Sciences,

University of Indone-

sia, Depok, West Java,

16424, Indonesia

E-mail: supriyanto.p@sci.

ui.ac.id

Received: 7 April 2020

Accepted: 18 June 2020 
In theory, dose calculation is simplified due to reduced head scatter, less off-axis softening, lower electron contamination, and lower leaf transmission on off-axis distance [1].

Another issue of TPS beam modelling was inhomogeneity correction. AAA, which has been implemented commercially to calculate dose distribution and embedded in Eclipse TPS was found debatable to calculate the absorbed dose in different density, especially when facing a low-density material that underestimated dose in a range of $8 \%-45 \%$ [2]. Inhomogeneity correction was needed for lung equivalent density of the regular field size of Linac [3]. The degradation of the dose was found when increasing the thickness of the air gap and larger field sizes were reported by Robinson et al, [4]. Aarup et al, (2009) studied the inhomogeneity effect of $15 \mathrm{~cm}$ lung densities on various dose algorithms such as AAA, pencil beam convolution (PBC), and collapse cone convolution (CCC). They found that $\mathrm{PBC}$ was inaccurate due to inflating lung volume and AAA was a good agreement to Monte Carlo (MC) if the density was $\geq 0.2 \mathrm{~g} / \mathrm{cm}^{3}$. On the other hand, the CCC algorithm was the best matched to $\mathrm{MC}$ of all algorithms [5].

Besides, the head and neck are parts of the human body containing cavities with lowdensity or air-filled material such as the trachea and nasal cavity. The cavities were less dense and smaller than the lung. These cavities became one of the main challenges of radiation cancer treatment of the head and neck area [6-8]. Moreover, the advanced technique of radiotherapy, either IMRT or Stereotactic Body Radiation Therapy (SBRT) were using a small beamlet of the multi-leaf collimator or small field size irradiation. Several authors have published the results of dose calculations in small fields for low-density materials, both measurements, and simulations. [9-11]. It had to be mentioned that the direct measurement of small field irradiation was difficult to be handled [12]. In order to reduce the error, MC simulation became an excellent method to ob- tain the absorbed dose on small field irradiation. However, several studies have reported the dose difference on TPS of the impact of low-density material either for regular or small field irradiation, no specific reports addressed small field FFF beams on small air-gap thickness so far. This study aimed to evaluate the effect of air-gap inhomogeneity dose calculation of FFF small field irradiation. MC simulation was employed to study the characteristics of the FFF beam in the heterogeneity region. Virtual phantoms of $0,2,4,6,8,10$, and 15 $\mathrm{mm}$ air-gaps were built. We did the simulation at $1 \times 1,2 \times 2,3 \times 3,4 \times 4 \mathrm{~cm}^{2}$ field sizes of $6 \mathrm{MV}$ FFF photon irradiation. The dose ratio of airgaps to tissue-equivalent was calculated both in AAA and MC. Moreover, we evaluated the planned dose distribution of head and neck cancer case based on the Monte Carlo simulation.

\section{Material and Methods}

In this experimental and Monte Carlo (MC) simulation study, we performed the dose calculation TPS, which implemented AAA algorithm at Pasar Minggu Regional General Hospital, Indonesia. The calculation was done at 2 $\mathrm{mm}$ dose grid inside the dimension of longitudinal $\times$ lateral $\times$ vertical direction of $30 \times 20$ $\times 16 \mathrm{~cm}^{3}$ virtual phantom for open small field and patient $\mathrm{CT}$ image for IMRT irradiation. Furthermore, the EGSnrc Monte Carlo code was employed to do the same parameter as a comparison of planned dose.

\section{Phantom/patient image}

We generated virtual phantom using MATLAB code, which was then saved in DICOM format. The scheme of the MC simulation on the phantom is displayed in Figure 1. Overall, the phantom design was divided into three regions, i.e. surface, air-gap, and after the airgap. The surface was conducted in a $2.67 \mathrm{~cm}$ tissue-equivalent density. The air-gap is a simulated area with various thickness of air density. We set the air-gap to $0,2,4,6,8,10$, and 15 


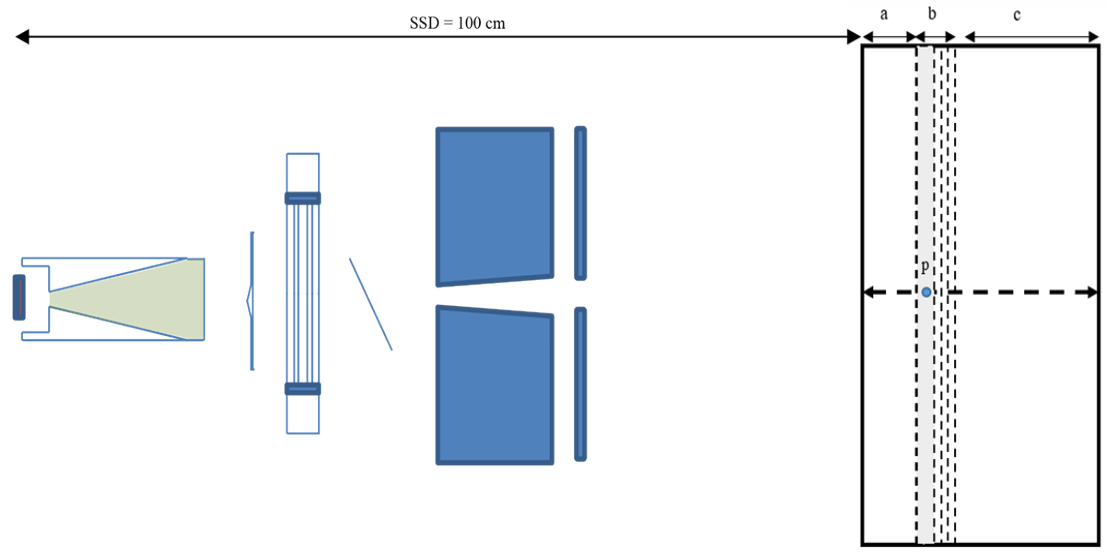

Figure 1: Flattening filter free (FFF) head Linac and MATLAB-based virtual phantom design of air gap in lateral view. Notation of $a, b$, and $c$ is $2.67 \mathrm{~cm}$ surface, various air-gap thickness, and after secondary build-up area, respectively. A thick vertical dashed line is the area of interest in this study where point $p$ is respected to the $1.4 \mathrm{~mm}$ inside the phantom to evaluate the backscatter factor calculation.

mm thickness in our work. A MATLAB image structure was constructed using unsigned integer 16-bit (uint 16), which had a pixel value $2^{\mathrm{n}}-1$ with $\mathrm{n}$ from 0 to 16 . Since the pixel value and Hounsfield Unit (HU) had a linear correlation [13], we defined 0-pixel value equals to $-1000 \mathrm{HU}$. The CT numbers inserted were 134 $\mathrm{HU} \approx 1.134 \mathrm{~g} / \mathrm{cm}^{3}$ and $-976 \mathrm{HU} \approx 0.024 \mathrm{~g} / \mathrm{cm}^{3}$ for tissue-equivalent and air, respectively. The voxel size of the virtual phantom was $0.14 \times$ $0.11 \times 0.11 \mathrm{~cm}^{3}$.

On the other hand, we performed the IMRT left parotid case on the patient CT-scan image. The target volume was next to the oral cavity, which is matched to our study design. Since the Monte Carlo and TPS dose calculation is based on electron density, we extracted the electron density from CT number during the commissioning of the Linac machine. Furthermore, the CT image was reconstructed with voxel dimension of $0.10 \times 0.10 \times 0.10 \mathrm{~cm}^{3}$.

Besides, the EGSnrc needed another format of the image called egsphant. The DICOM file was converted into egsphant file using CT-create, which was a user code to build the readable image from $\mathrm{CT}$. In order to obtain the same voxel size as DICOM file, the phantom dimension at egsphant was cropped to $17 \times 14$ $\times 14 \mathrm{~cm}^{3}$ symmetrically from the central axis.

TPS calculation and Monte Carlo simulation

We used an analytical anisotropic algorithm (AAA), implemented in the Eclipse planning system to obtain the dose distribution inside the phantom. AAA is an advanced algorithm based on a pencil beam method, which iterates the beam straight vertically on the z-axis and then superposition it laterally. The irradiation technique was source to surface distance (SSD), $100 \mathrm{~cm}$ open field at $0^{\circ}$ gantry rotation with a variation of small field sizes. In this work, we retract the multileaf collimator (MLC). On other words, we shaped the radiation fields to $1 \times 1,2 \times 2,3 \times 3,4 \times 4 \mathrm{~cm}^{2}$ on the virtual phantom surface using jaws. The selected energy was $6 \mathrm{MV}$ with FFF mode and normalization to the depth maximum. For the IMRT case, we applied 5 gantry angles method with isocenter on the middle of the planning target volume (PTV). The isocenter was also chosen to be a normalization point in our study. The irradiation field sizes is depended on the PTV's beam eye view and the MLC is used to modulate the intensity inside the target volume. 
We employed EGSnrc Monte Carlo simulation with the similar techniques. The simulation itself consisted of two main programs. First, the particles were simulated inside the head of FFF Linac, whose structure generated from the previous study [14]. The particle source was electrons with the kinetic energy of $6.2 \mathrm{MeV}$ and a full-width half-maximum of $0.2 \mathrm{~cm}$. These particles bombarded a tungsten target and produced the photon beams radiation. The photon would penetrate and attenuate at primary collimator, vacuum glass, filter, monitor chamber, mirror, jaws, and MLCs. The flattening filter in this study was replaced by a filter called FakeBeam filter [15], which has been investigated in our previous work for regular and small field irradiation [16]. The MLC movement parameters were obtained from the MLC sequence of the TPS for the IMRT case. We collected the phase space file, stored the characteristics of the particles after the MLCs structure. The phase space file then became the source in the second term of Monte Carlo simulation. Second, Monte Carlo simulated the interactions inside the virtual phantom and patient images. The particles simulated in this work were $4 \times 10^{8}$ histories, and the simulation would be stopped when the energy of photon and electron was 0.01 and $0.521 \mathrm{MeV}$, respectively. In order to reduce the simulation time, we used direct bremsstrahlung splitting with a $20 \mathrm{~cm}$ spread radius. Furthermore, we exported the dose distribution inside the virtual phantom and patient images for both TPS and Monte Carlo simulation.

The focus of the interest of this study was the radiation interaction in the effect of the small air-gaps. The percentage dose differences (PDD) of each field sizes and dose distribution on the patient's axial view were collected and then compared between TPS calculation and Monte Carlo simulation. A zero air-gap or homogeneous virtual phantom became a standard normalization point of all the air-gap variation at each field size. Since the Monte Carlo became an ideal dose distribution meth- od in this study, the calculated doses from TPS were adjusted to simulated doses of Monte Carlo in order to reduce the error at the heterogeneous material. Equation 1 described this adjusted definition:

$$
D_{T P S, p}^{*}=D_{T P S, p} \times \frac{D_{M C, p, h}}{D_{T P S, p, h}}
$$

Where $D^{*}{ }_{T P S, p}$ and $D_{T P S, p}$ were TPS calculated doses with adjusted and nonadjusted point $p$ in a heterogeneity phantom, respectively. $D_{M C, p, h}$ and $D_{T P S, p, h}$ were Monte Carlo simulated dose and TPS calculated dose at a point $\mathrm{p}$ in a homogeny phantom. Equation 1 was necessary if the dose difference between Monte Carlo and TPS was beyond $2 \%$ [9]. The dose difference $(\% D)$ itself followed the Equation 2.

$$
\% D=\frac{D_{M C, p}-D_{T P S, p}^{*}}{D_{M C, p}} \times 100 \%
$$

Where $D_{M C, p}$ was the Monte Carlo simulated dose at a point $p$ in a heterogeneity phantom. Moreover, this study looked for the correction needed of TPS dose calculation as an impacted of perturbation of the thin air gap inside the phantom. The dose correction factor (DCF) between Monte Carlo and TPS was then compared inside the air-gap after the secondary build-up region beyond the air-gap. DCF followed Equation 3.

$$
D C F=\frac{D_{T P S, p}^{*}}{D_{T P S, p, h}} \times \frac{D_{M C, p, h}}{D_{M C, p}}
$$

\section{Results}

The evaluation of dose distribution was firstly carried out on the homogeneous phantom in order to check whether it needed a dose adjustment or not in the later analysis. The percentage depth dose (PDD) at the central axis of the homogeneous phantom was plotted based on both TPS calculation and MC simulation for all field sizes. We normalized the PDDs at the depth maximum and found that the dose distributions of TPS calculation before adjustment were smaller than the MC calculation. The maximum depth of TPS calculation and MC 
simulation was at 1.26 and $1.12 \mathrm{~cm}$ for all field sizes, respectively. In other words, there was a $0.14 \mathrm{~cm}$ shift of maximum doses. Since the shift was small enough, we did not correct the shifting of these dose distributions.

Moreover, the dose difference at each depth was generated between TPS and MC. The dose difference maximum for $1 \times 1,2 \times 2,3 \times 3$, and $4 \times 4 \mathrm{~cm}^{2}$ was $-7.57,-8.43,-8.79$, and $-6.61 \%$, respectively. The dose differences on the deeper area was higher than the shallow one. We assumed that the TPS calculation underestimated the dose because of the lack of scattering iteration inside the AAA method, especially for small field sizes beam irradiation. Based on the results mentioned above, we adjusted the TPS calculation of homogenous phantom to $\mathrm{MC}$ simulation so that the TPS calculation hereafter means TPS calculation after adjustment.

\section{Inside the air-gaps}

The TPS calculation and MC simulation were done at a various air-gap thickness of virtual homogeneous phantoms. Since we repeated the calculation and simulation six times, some points of depth were evaluated 7-i times where $i$ was the iteration of thinnest to thickest meaning $i=1,2,3,4,5,6$ for 2 , $4,6,8,10$, and $15 \mathrm{~mm}$ air-gap thickness, respectively. The result of doses was quite similar for the repetition with difference air-gaps phantom for TPS calculation with a standard error of $0.12 \%$. However, Monte Carlo simulation showed a higher dose when the point of interest was near the end of air-gaps, as shown in Table 1.

Furthermore, the discrepancy in absorbed dose inside the air-gap for TPS calculation and $\mathrm{MC}$ simulation was very high. Figures $2 \mathrm{a}$ and $b$ show the PDD of tissue-equivalent and air material densities concerning to describe the differences absorbed dose from MC simulation and adjusted TPS calculation. The evaluation was done inside the most substantial airgap phantom which started at $2.80 \mathrm{~cm}$ to 4.07 $\mathrm{cm}$ below the phantom surface. For TPS dose evaluation on open small fields irradiation, we calculated the dose difference of the TPS PDDs to MC PDDs, as described in Figure 3.

A huge dose difference was found at $1 \times 1 \mathrm{~cm}^{2}$ field size which had a deviation of up to $134 \%$. However, the dose difference became low with an increasing field size so that $4 \times 4 \mathrm{~cm}^{2}$ had a maximum deviation of $23.5 \%$. However, the dose difference was still out of the tolerance limit as the results indicated that AAA calculation was significantly improved at larger field sizes when facing a very low-density material.

We found that both MC simulation and TPS calculations had a linear correlation between a step-up dose ratios with an increment of field sizes, as seen in Figure 4. These ratios were agreed with a linear trend line of $y=a x+c$ only for small field irradiation up to $4 \times 4 \mathrm{~cm}^{2}$. We found that the slope for both AAA and MC

Table 1: Percentage dose of Monte Carlo (MC) simulation and treatment planning system (TPS) calculation inside the air gap at $1.4 \mathrm{~mm}$ (near the end of air-gap of $2 \mathrm{~mm}$ thickness) below the boundary.

\begin{tabular}{|c|c|c|c|c|c|c|c|c|}
\hline Air-gap & \multicolumn{4}{|c|}{ Monte Carlo simulation dose (\%) } & \multicolumn{4}{|c|}{ Treatment planning system calculation dose (\%) } \\
\hline Thickness (mm) & $1 \times 1 \mathrm{~cm}^{2}$ & $2 \times 2 \mathrm{~cm}^{2}$ & $3 \times 3 \mathrm{~cm}^{2}$ & $4 \times 4 \mathrm{~cm}^{2}$ & $1 \times 1 \mathrm{~cm}^{2}$ & $2 \times 2 \mathrm{~cm}^{2}$ & $3 \times 3 \mathrm{~cm}^{2}$ & $4 \times 4 \mathrm{~cm}^{2}$ \\
\hline 2 & 69.20 & 78.12 & 81.53 & 82.36 & 85.96 & 88.64 & 90.49 & 90.32 \\
\hline 4 & 66.94 & 75.32 & 78.03 & 80.32 & 85.80 & 88.77 & 90.48 & 90.09 \\
\hline 6 & 66.36 & 73.92 & 77.03 & 81.86 & 86.07 & 88.51 & 90.26 & 90.30 \\
\hline 8 & 65.98 & 73.76 & 77.21 & 80.55 & 86.19 & 88.57 & 90.48 & 90.21 \\
\hline 10 & 66.50 & 72.87 & 76.93 & 81.09 & 86.13 & 88.59 & 90.48 & 90.30 \\
\hline 15 & 65.71 & 72.22 & 76.06 & 76.35 & 86.01 & 88.79 & 90.21 & 90.32 \\
\hline
\end{tabular}




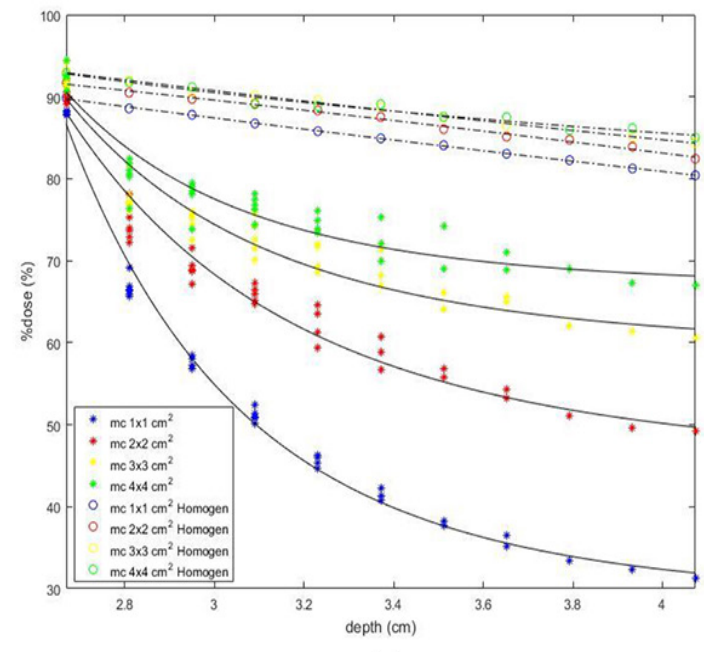

(a)

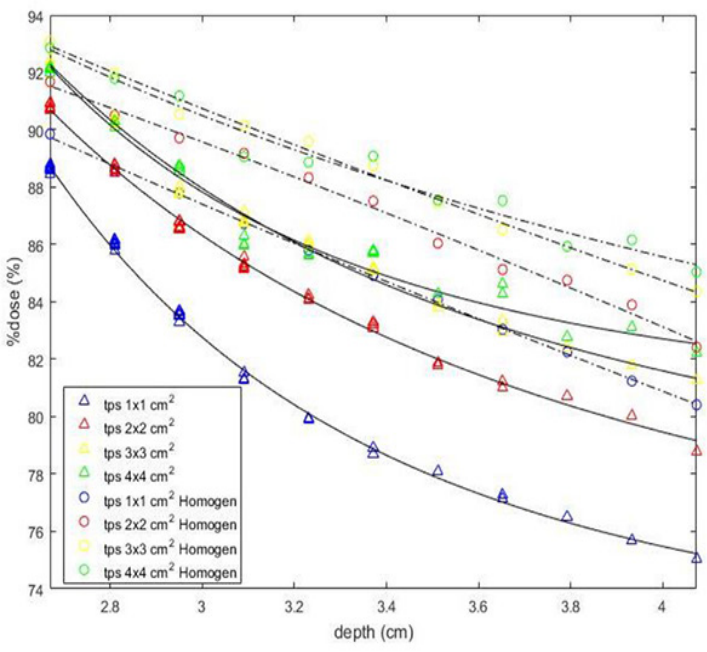

(b)

Figure 2: The percentage dose differences (PDD) of homogeneous and inhomogeneous phantom for $1 \times 1,2 \times 2,3 \times 3$, and $4 \times 4 \mathrm{~cm}^{2}$ (a) Monte Carlo simulation and (b) Treatment planning system (TPS) calculation.

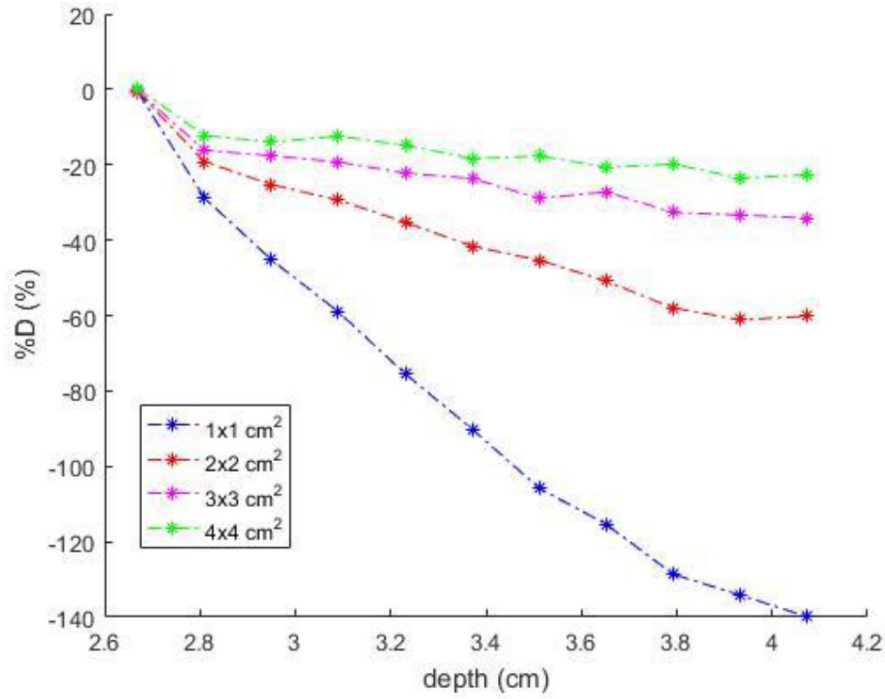

Figure 3: Dose difference between treatment planning system (TPS) calculation and Monte Carlo $(\mathrm{MC})$ simulation inside the air gap at $1 \times 1$ (blue), $2 \times 2$ (red), $3 \times 3$ (magenta), and $4 \times 4$ (green) $\mathrm{cm}^{2}$ field sizes.

was slightly differenced, i.e. $-0.198 \pm 0.001$ and $-0.161 \pm 0.014$, respectively. Based on the dose difference within the air-gap density, we obtained the average dose correction factor of this area, as seen in Figure 5. The DCF is reversible to the dose difference and the largest DCF is found on the smallest fields in this study. However, DCF moved to unity with larger field size. On the $4 \times 4 \mathrm{~cm}^{2}$ field size, the correction was independent of the air-gaps thickness.

\section{After secondary build-up}

We evaluated the absorbed dose at tissue- 


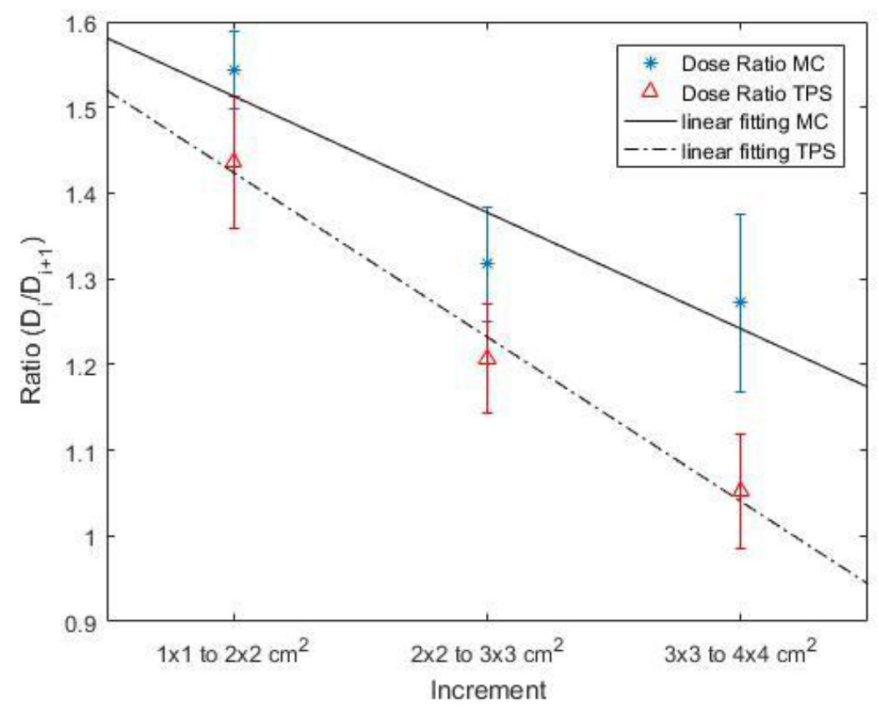

Figure 4: The step-up dose ratio with an increment of field sizes on small field irradiation. The red triangle and blue star belong to treatment planning system (TPS) and Monte Carlo (MC) dose ratio, respectively.

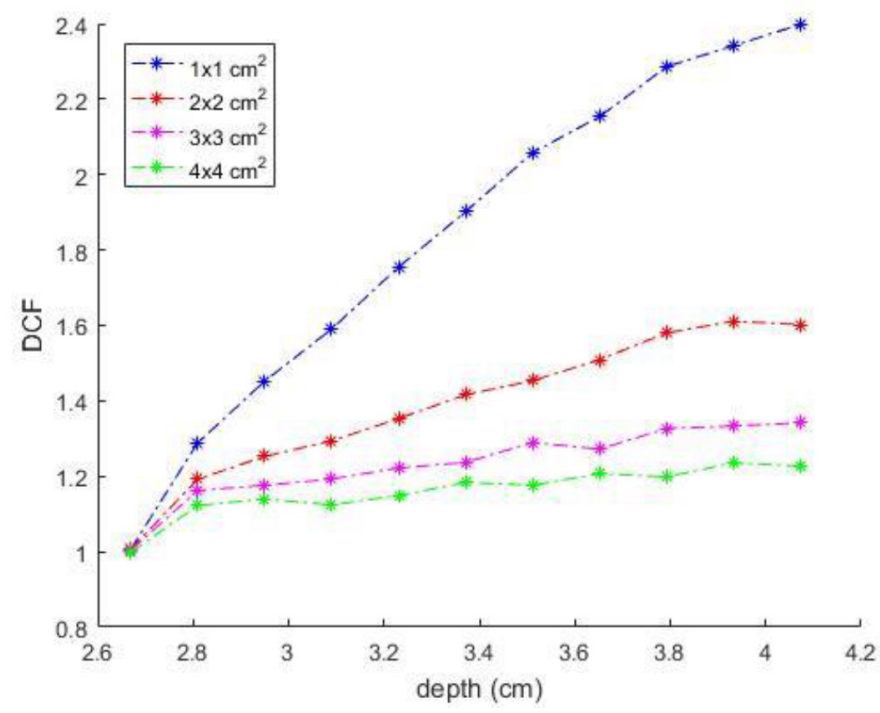

Figure 5: Dose correction factor (DCF) of treatment planning system (TPS) calculation in respect to Monte Carlo (MC) simulation inside the air-gap density at $1 \times 1$ (blue), $2 \times 2$ (red), $3 \times 3$ (magenta), and $4 \times 4$ (green) $\mathrm{cm}^{2}$ field sizes.

equivalent material after the secondary buildup area as a perturbation effect due to charged particle equilibrium loss inside the air-gap. The average dose difference between homogeneous and inhomogeneous phantom at $1 \times 1$, $2 \times 2,3 \times 3$, and $4 \times 4 \mathrm{~cm}^{2}$ field sizes for both MC simulation and TPS calculation were shown in
Figures $6 a$ and $b$, respectively. Those graphs describe that dose difference after secondary build-up in heterogeneous would be higher than homogeneous phantom with an increase of air-gap thickness while decreasing with larger field size.

On the other hand, we also compared the 


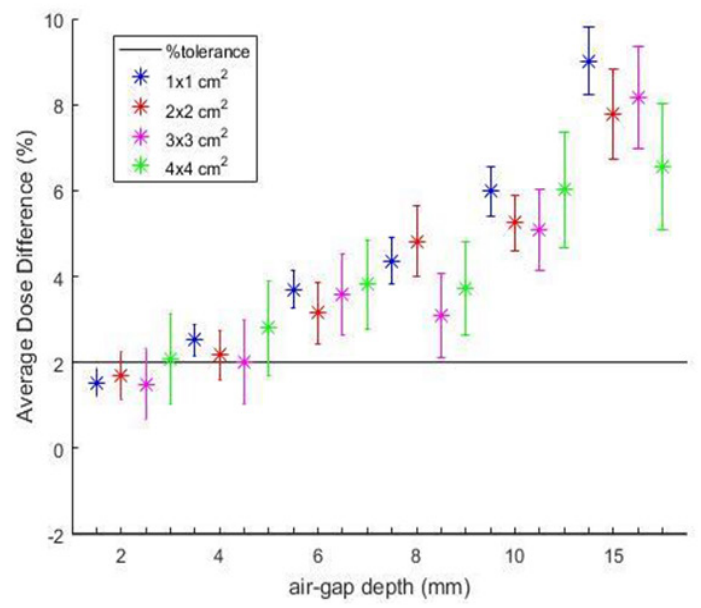

(a)

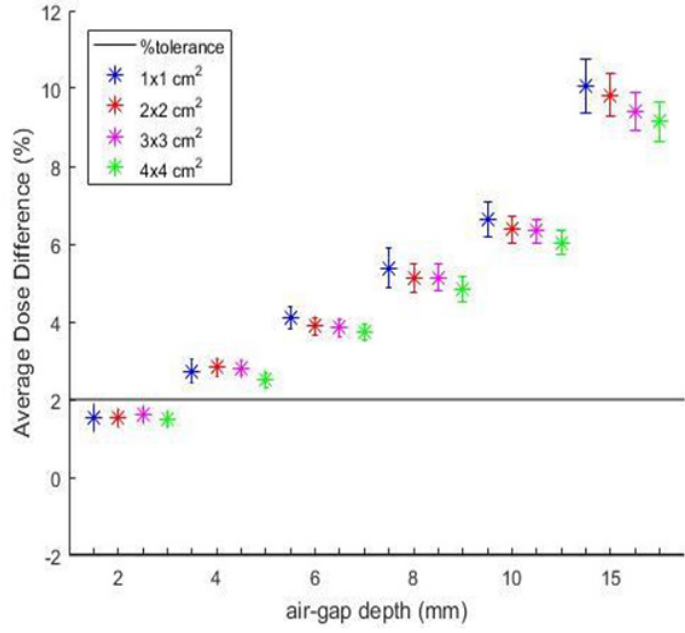

(b)

Figure 6: Average dose difference between homogeneous and inhomogeneous phantom for various air-gap thickness at $1 \times 1$ (blue), $2 \times 2$ (red), $3 \times 3$ (magenta), and $4 \times 4$ (green) $\mathrm{cm}^{2}$ for (a) Monte Carlo simulation and (b) Treatment planning system calculation after the secondary build-up region.

absorbed dose between MC and TPS for the tissue-equivalent material after the air-gap. Generally, the dose difference of TPS calculation and $\mathrm{MC}$ simulation was agreed within $\pm 2 \%$ of tolerance. Henceforth, the dose differences tended to move out of tolerance for larger air-gap and more extensive field size. In this study, we obtained the average dose difference of $2.45 \pm 1.19 \%$ for $4 \times 4 \mathrm{~cm}^{2}$ field size and 1.5 cm air-gap. Based on these data, the AAA calculation was affected by the density applied to the system especially at very low-density material, which could be inaccurate absorbed dose estimation either for regular beam or FFF beam. As a result, DCF is preferably implemented to the AAA calculation even after the secondary build-up region. Figure 7 shows the DCF for all small field sizes in this research

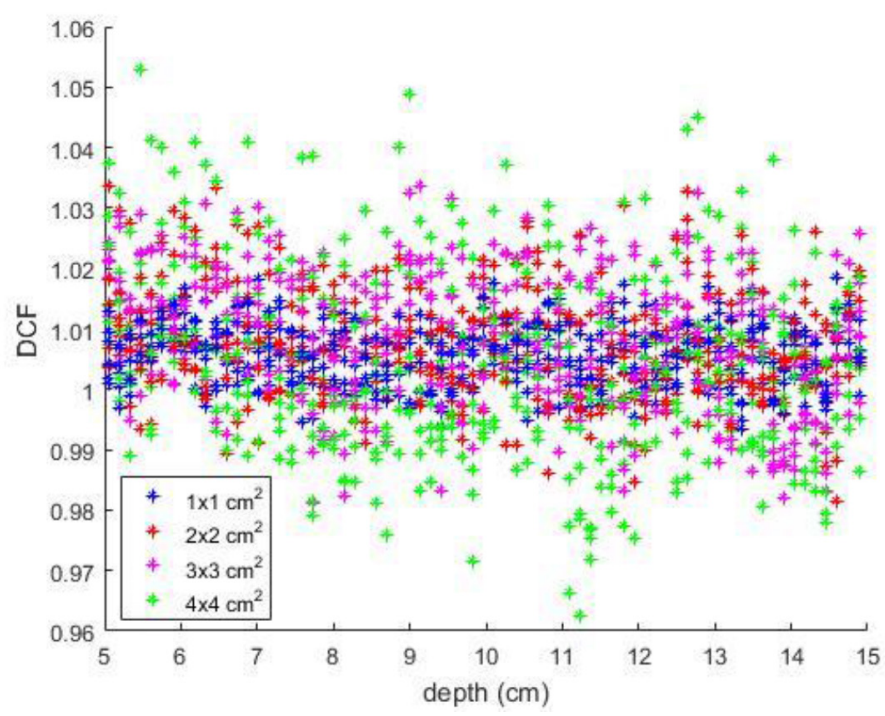

Figure 7: Dose correction factor (DCF) of treatment planning system (TPS) calculation to Monte Carlo (MC) as benchmark after secondary build-up area at $1 \times 1$ (blue), $2 \times 2$ (red), $3 \times 3$ (magenta), and $4 \times 4$ (green) $\mathrm{cm}^{2}$ field sizes for all air-gap variation. 
through central axis depth below the virtual phantom surface. The DCF results indicate that it should be aware of using larger field sizes because the DCF tended to leave a unity, whereas it approached one inside the air-gap.

\section{IMRT head and neck case}

We investigated the effect of small air-gap on the real patient $\mathrm{CT}$ image for the clinical implementation of FFF 6 MV Linac. Left parotid was delineated as a PTV in our study. The dose distribution on an axial CT image slice is evaluated using a simple dose difference inside the patient's body. We also compared the dose distribution inside the PTV, which margin actually overreach to the oral cavity. The comparison of TPS calculation and MC simulation on patient CT image is seen in Figure 8. We evaluated one patient's image slice which intersect the isocenter and found that TPS cal- culation is better than the MC simulation on the PTV with relative average dose of $98.31 \pm$ $1.76 \%$ and $96.70 \pm 10.72 \%$ for TPS and MC simulation, respectively. A non-conformal dose distribution on the MC simulation is because of the PTV included a small portion of oral cavity.

\section{Discussion}

The study of comparison between TPS calculation, which especially focused on AAA, and MC simulation for $6 \mathrm{MV}$ FFF Linac showed a perturbation effect because of small air-gap on the radiation track. Even though the maximum energy of FFF Linac in our study is around $6 \mathrm{MeV}$, the probable energy is on the low energy due to the beam hardening effect of FFF design. On the other word, the dose was dominantly contributed from the primary beam and forward scattered of particles. How- (a)
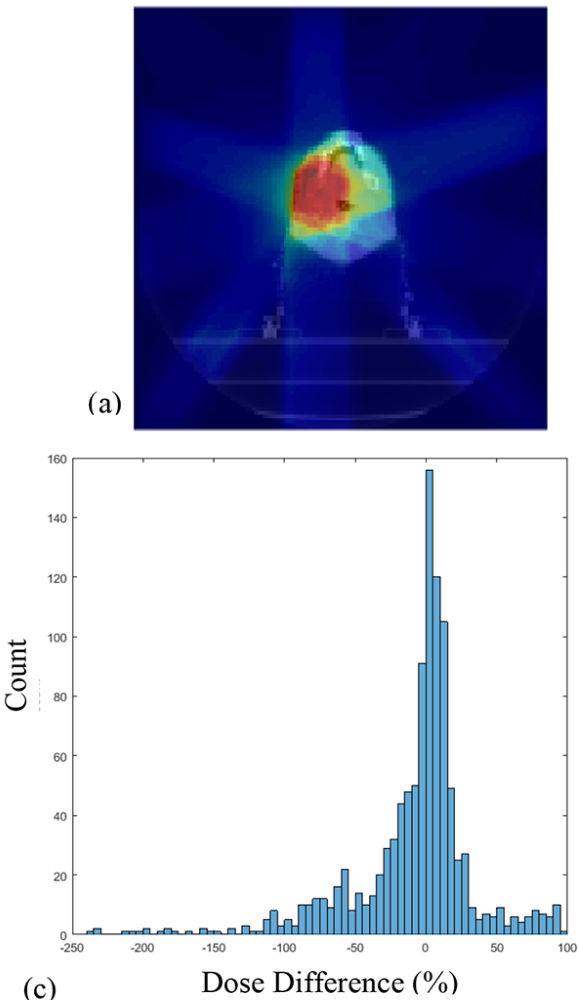

(b)
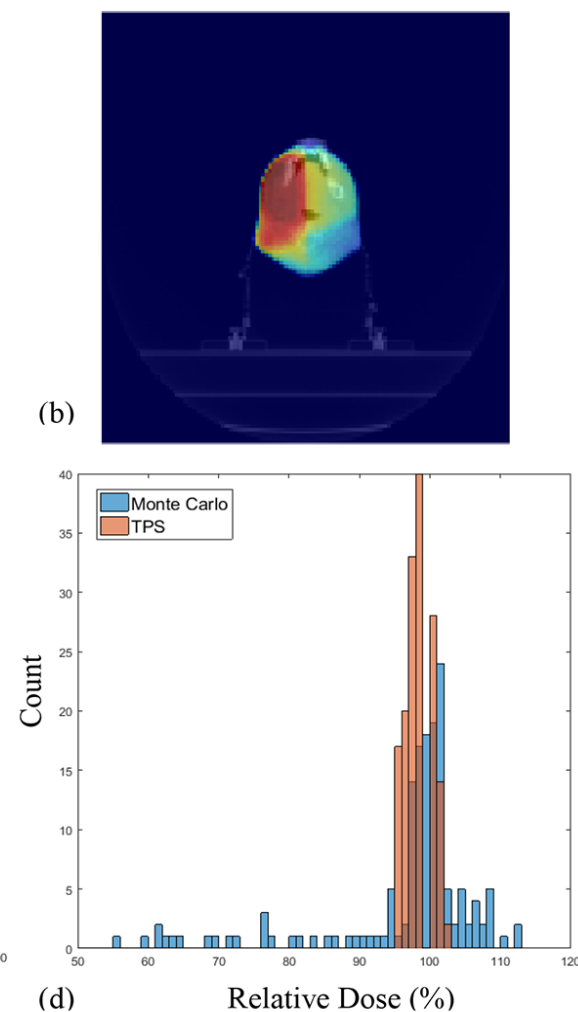

Figure 8: Evaluation of treatment planning system (TPS) and Monte Carlo (MC) on left parotid case, (a) dose distribution of MC, (b) dose distribution of TPS, (c) dose difference histogram of TPS and MC inside the patient's body, and (d) relative dose histogram of TPS and MC at planning target volume (PTV) 
ever, TPS seemed not to count the backscatter phenomena, which could be impacted by the absorbed dose. Table 1 shows the difference dose because of this effect at $2.80 \mathrm{~cm}$ depth (1.4 $\mathrm{mm}$ below the tissue-equivalent boundary) for all small field sizes. The back scatter effect is prominent for bigger field size according to the $\mathrm{MC}$ simulation, but it is not clearly shown for TPS calculation at $3 \times 3$ and $4 \times 4 \mathrm{~cm}^{2}$ field sizes. Tan et al., (2014) investigated the common algorithm of photon dose calculation from regular Linac machine. Their finding was agreed with our result that almost there is the lack of back scatter consideration on AAA algorithm [17].

Inside the air-gap phantom, the absorbed dose seemed had a correlation with increasing depth in this region for both MC simulation and TPS calculation. We operated a power equation of $y=a x^{b}+c$ to fit the data of PDD inside the air-gap region where the mean coefficienta,b and c were $(2.05 \pm 1.69) \times 10^{4}$, $-6.20 \pm 0.74$, and $49.91 \pm 16.84$ for all field sizes, respectively. The Monte Carlo result shows a functional similarity with Jones and Das, who used the small field of a regular Linac that in the region up to $1.5 \mathrm{~cm}$ below the boundary or build down area was to decrease exponentially. The extrapolation of the perturbation factor between simulated dose in inhomogeneous to homogeneous phantom was around 0.35 at near-zero density at their result of $1 \times 1 \mathrm{~cm}^{2}$ field size correlated with our results, which achieved about 0.39 [9]. Based on these results, FFF and regular Linac, differing on the flattening filter, had deviation slightly on the perturbation effect of small field size irradiation.

On the other hand, the perturbation effect of low-density seemed not to affect the absorbed dose of the AAA calculation. These results were also justified by Aarup et al., who studied the effected low-densities of specific algorithms at $2.8 \mathrm{~cm}$ diameter field size on regular Linac. Their results show that the AAA algorithm clinically feasible for the density of $\geq$
$0.2 \mathrm{~g} / \mathrm{cm}^{3}$ [5]. Moreover, the PDDs were found higher at $3 \times 3$ and $4 \times 4 \mathrm{~cm}^{2}$ field sizes than the $1 \times 1 \mathrm{~cm}^{2}$ field size in tissue-equivalent density. This data indicated that the contribution of field size to absorbed dose on the central axis was significantly higher for small field FFF irradiation rather than the density of absorbance medium in AAA calculation method. Besides, very high dose difference was found inside air gap. It was agreed by Muralidhar et al., who studied the absorbed dose calculation of AAA based-on six difference densities respected to Acuros calculation for the FFF Linac beam at $30 \times 30 \mathrm{~cm}^{2}$. The results showed that the difference of AAA was only $2.6 \%$ at -993 HU compared to Acuros calculation [18].

We calculated the dose correction by applying equation 3 inside the air-gap and the results was shown on Figure 5. For the smallest field size, the DCF was up to 2.4 and decreased with bigger field size. This trend was agreed with Jones and Das (2005), who found the DCF for several algorithms for regular Linac [9]. The DCF was necessary needed at the smallest field size and thick air gap. Furthermore, the DCF became more stable along with the depth of air-gap for larger field size and the DCF might be not necessary when a large field size was used for the irradiation with small air-gap thickness. However, we had to recognize that a small air-gap within the patient body such as the trachea, oral, and nasal cavities, would change the physical properties of the beam especially for the FFF Linac beam due to the high gradient change of densities.

The perturbation effect as an impact of the air-gap became our field of interest because most of the head and neck target is at the tissue surround by cavities. Both MC simulation and TPS calculation showed that the perturbation effect at $2 \mathrm{~mm}$ air-gap thickness could be neglected because dose differences of all small field sizes in this area were inside the tolerance level of $2 \%$. Charles et al., who did OSLD simulation for regular Linac at small field size and $0.5 \mathrm{~mm}$ air-gap, agreed with this 
result. They found that the dose difference was reduced from -7.8 to $-0.8 \%$ for 6 to $42 \mathrm{~mm}$ field sizes [11]. The extrapolation parameter of this study was done by Robinson et al., which agreed with our findings. They used a $5 \times 5 \mathrm{~cm}^{2}$ field size for two air-gap variations on the FFF Linac beam and found the dose difference between measured and TPS was $3.1 \%$ and $-2.4 \%$ for 7.5 and $15 \mathrm{~cm}$, respectively. Bush et al., showed the dose difference between AAA and MC simulation on regular Linac for $4 \times 4 \mathrm{~cm}^{2}$ field size after a secondary build-up of $10 \mathrm{~cm}$ air-gap with $0.001 \mathrm{~g} / \mathrm{cm}^{3}$ density was around $5-10 \%[2,4]$.

Clinical treatment of FFF Linac photon beam is commonly implemented on head and neck cancers. We compared the dose distribution of TPS calculation to MC simulation on left parotid carcinoma, which PTV was drawn over the oral cavity as an indicator of small air-gap inside the patient's body. It can be seen in Figures $8 \mathrm{a}$ and $\mathrm{b}$ that the dose distribution inside the body is visually difference between MC and TPS. In our MC simulation, the absorbed dose of photon beam is low at the back end of the patient's skin. This effect is as the photon beam of gantry 0 is facing the high density of teeth and less contribution on the back of the patient. However, we found that skin dose on the MC simulation was higher than TPS calculation. Figure $8 \mathrm{c}$ shows the cumulative histogram of dose difference between TPS and MC inside the patient's body. The result very varying with the mode of the dose difference is within the range of $0 \sim 5 \%$. The histogram can be dived into three categories. The negative deviation was due to the patient cavity in this case; this effect showed that MC simulation has a lower absorbed dose than TPS simulation, thus, the negative value is generated. The high dose difference was conducted because of the difference dose imparted on the left skin of the patient. The relatively small dose difference is on the tumor target. Furthermore, we investigated only the patient's PTV and obtained the dose distribution histogram as shown in Figure 8d. Even though the area is the oral cavity, where the low-density material is presented, the dose distribution of TPS is conformed to $100 \%$ of prescribed dose. On the other hand, the dose result on MC simulation is distributed nearly to $50 \%$ as an impacted of air-gap on the patient's mouth.

\section{Conclusion}

TPS, which utilized AAA dose calculation at small field sizes of eclipse TPS of $6 \mathrm{MV} \mathrm{FFF}$ Linac photon beam in the virtual inhomogeneous phantom, was assessed by comparing to EGSnrc Monte Carlo as a benchmark. The investigation was divided into two areas, i.e. inside the air-gap and after secondary buildup. Inside the air-gap, TPS calculation overestimated the absorbed dose compared to MC simulation. We found that the dose difference was up to $134 \%$ for $15 \mathrm{~mm}$ air-gap at $1 \times 1 \mathrm{~cm}^{2}$, and the decreasing dose ratio was linear with increasing field size. Moreover, the TPS calculation seemed not to include the backscatter contribution to the algorithm. After the airgap boundary, the perturbation effect at $2 \mathrm{~mm}$ air-gap thickness could be neglected for all small field sizes. Besides, the dose difference between TPS calculation and MC simulation generally was within the tolerance level of $2 \%$ in this study. The results show that DCF inside the air-gap was moving to one with increasing field size. Contradictory, the DCF was reversibly out from unity with increasing field size. On the IMRT case, which PTV overreached to the air-gap, the dose distribution of AAA calculation could be led to overestimate, however, the dose difference after the air-gap was reduce because of the contribution of others gantry angles.

\section{Acknowledgment}

This study was supported by Pasar Minggu Regional General Hospital and University Indonesia Research Grant (PIT9) with contract number NKB-0034/UN2.R3.1/ HKP.05.00/2019. 
Azzi A., Ryangga D., Pawiro S. A.

\section{Conflict of Interest}

None

\section{References}

1. Kragl G, Albrich D, Georg D. Radiation therapy with unflattened photon beams: dosimetric accuracy of advanced dose calculation algorithms. Radiother Oncol. 2011;100(3):417-23. doi: 10.1016/j. radonc.2011.09.001. PubMed PMID: 21945857.

2. Bush K, Gagne IM, Zavgorodni S, Ansbacher W, Beckham W. Dosimetric validation of Acuros XB with Monte Carlo methods for photon dose calculations. Med Phys. 2011;38(4):2208-21. doi: 10.1118/1.3567146. PubMed PMID: 21626955.

3. Teke T, Duzenli C, Bergman A, Viel F, Atwal P, Gete E. Monte Carlo validation of the TrueBeam 10XFFF phase-space files for applications in lung SABR. Med Phys. 2015;42(12):6863-74. doi: 10.1118/1.4935144. PubMed PMID: 26632043.

4. Robinson J, Opp D, Zhang G, Feygelman V. Evaluation of inhomogeneity correction factors for $6 \mathrm{MV}$ flattening filter-free beams with brass compensators. J Appl Clin Med Phys. 2013;14(3):3990. doi: 10.1120/jacmp.v14i3.3990. PubMed PMID: 23652238. PubMed PMCID: PMC5714406.

5. Aarup LR, Nahum AE, Zacharatou C, et al. The effect of different lung densities on the accuracy of various radiotherapy dose calculation methods: implications for tumour coverage. Radiother Oncol. 2009;91(3):405-14. doi: 10.1016/j. radonc.2009.01.008. PubMed PMID: 19297051.

6. Seif F, Bayatiani MR, Hamidi S, Kargaran M. Investigating the Effect of Air Cavities of Sinuses on the Radiotherapy Dose Distribution Using Monte Carlo Method. J Biomed Phys Eng. 2019;9(1):1216. doi: 10.31661/JBPE.V9I1FEB.1046. PubMed PMID: 30881941. PubMed PMCID: PMC6409366.

7. Singh N, Sharma SD, Painuly NK, et al. Underdosing of the maxillary sinus for small fields used in newer radiotherapy techniques: Comparison of thermoluminescent dosimeter and Monte Carlo data. J Cancer Res Ther. 2018;14(2):351-6. doi: 10.4103/0973-1482.183195. PubMed PMID: 29516918.

8. Lu JY, Zheng J, Zhang WZ, Huang BT. Flattening Filter-Free Beams in Intensity-Modulated Radiotherapy and Volumetric Modulated Arc Therapy for Sinonasal Cancer. PLoS One. 2016;11(1):e0146604. doi: 10.1371/journal.pone.0146604. PubMed PMID: 26734731. PubMed PMCID: PMC4703390.

9. Jones AO, Das IJ. Comparison of inhomogeneity correction algorithms in small photon fields. Med Phys. 2005;32(3):766-76. doi: 10.1118/1.1861154.
PubMed PMID: 15839349.

10. Izewska J, Wesolowska P, Azangwe G, et al. Testing the methodology for dosimetry audit of heterogeneity corrections and small MLCshaped fields: Results of IAEA multi-center studies. Acta Oncol. 2016;55(7):909-16. doi: 10.3109/0284186X.2016.1139180. PubMed PMID: 26934916. PubMed PMCID: PMC4926790.

11. Charles PH, Crowe SB, Kairn T, et al. The effect of very small air gaps on small field dosimetry. Phys Med Biol. 2012;57(21):6947-60. doi: 10.1088/0031-9155/57/21/6947. PubMed PMID: 23044638.

12. Thwaites DI, Cranmer-Sargison G, Charles PH, Trapp JV. Measurement, modelling and reporting of small field output factors requires systematic approaches for accurate dosimetry. Physica Medica: Eur J Med Phys. 2014;30:e33-4. doi: 10.1016/j. ejmp.2014.07.107.

13. Razi T, Niknami M, Alavi Ghazani F. Relationship between Hounsfield Unit in CT Scan and Gray Scale in CBCT. J Dent Res Dent Clin Dent Prospects. 2014;8(2):107-10. doi: 10.5681/joddd.2014.019. PubMed PMID: 25093055. PubMed PMCID: PMC4120902.

14. Azzi A, Ryangga D, Pawiro SA. The characteristics of small field beam quality and output factor of 6 MV FFF. J Phys Conf Ser. 2019;1248(1):012056. doi: 10.1088/1742-6596/1248/1/012056.

15. Rodriguez M, Sempau J, Fogliata A, Cozzi L, Sauerwein W, Brualla L. A geometrical model for the Monte Carlo simulation of the TrueBeam linac. Phys Med Biol. 2015;60(11):N219-29. doi: 10.1088/0031-9155/60/11/N219. PubMed PMID: 25984796.

16. Pawiro SA, Azzi A, Soejoko DS. A Monte Carlo Study of Photon Beam Characteristics on Various Linear Accelerator Filters. J Biomed Phys Eng. 2020;10(5):613-22. doi: 10.31661/jbpe.v0i0.1192. PubMed PMID: 33134221. PubMed PMCID: PMC7557471.

17. Tan YI, Metwaly M, Glegg M, Baggarley S, Elliott $A$. Evaluation of six TPS algorithms in computing entrance and exit doses. J Appl Clin Med Phys. 2014;15(3):229-40. doi: 10.1120/jacmp. v15i3.4739. PubMed PMID: 24892349. PubMed PMCID: PMC5711058.

18. Muralidhar KR, Pangam S, Srinivas $P$, et al. A phantom study on the behavior of Acuros XB algorithm in flattening filter free photon beams. $J$ Med Phys. 2015;40(3):144-9. doi: 10.4103/09716203.165076. PubMed PMID: 26500400. PubMed PMCID: PMC4594383. 